\title{
Impact of time and work:rest ratio matched sprint interval training programmes on performance: A randomised controlled trial
}

\section{Abstract}

Objectives

The aim of this study was to examine the effects of a short training intervention using two repeated sprint protocols matched for total sprint duration and work:rest ratio.

\section{Design}

Randomised-controlled trial

\section{Methods}

Thirty physically active males were randomly allocated to one of two sprint training groups: a 6 second group, a 30 second group or a non-exercising control. The training groups were matched for work:rest ratio and total sprint time per session, and completed 6 training sessions over a 2-week period. Before and after the 2 week training period, participants completed a $\mathrm{VO}_{2 \max }$ test and a $10 \mathrm{~km}$ time trial on a cycle ergometer.

Results

Time trial performance increased significantly by $5.1 \%$ in $6 \mathrm{sec}(630 \pm 115 \mathrm{sec}$ to $598 \pm 92$ sec; $p<0.05)$ and $6.2 \%$ in $30 \mathrm{sec}(579 \pm 68 \mathrm{sec}$ to $543 \pm 85 \mathrm{sec} ; p<0.05)$ from baseline testing, but there was no significant change in the control group ( $p>0.05)$, and no significant difference between exercise groups ( $p>0.05)$. The 6 sec group increased peak power output by $9.0 \%$ (from $1092 \pm 263 \mathrm{~W}$ to $1181 \pm 248 \mathrm{~W} ; \mathrm{p}<0.05$ ) from sprint session 1 to 6 , and the 30 sec group by $20.0 \%(1041 \pm 161 \mathrm{~W}$ to $1237 \pm 159 \mathrm{~W} ; \mathrm{p}<0.05)$.

\section{Conclusions}

This study indicates that both 6 and 30 second bouts of repeated sprint exercise, matched for total sprint duration and W:R can improve athletic performance. 


\section{Introduction}

There has been renewed interest in the use of sprint interval training (SIT) as an exercise intervention in athletic, recreational and sedentary populations. ${ }^{1-2}$ Despite total exercise time being considerably lower in comparison with traditional endurance training approaches, similar increases in $\mathrm{VO}_{2 \max }$ (defined as the maximum rate at which an individual can take up and utilise oxygen), muscle oxidative capacity and exercise performance have been observed. ${ }^{3-7}$ This training approach is characterised by repeated bouts of relatively brief intermittent exercise, with an 'all-out' effort ${ }^{8}$, and may be more enjoyable than prolonged endurance training. ${ }^{9-10}$

Many studies looking into the effect of SIT have used a 30 second supramaximal exercise sprint bout with 4 minutes recovery $(1: 8$, work to rest ratio $(W: R))$, and have reported a range of central adaptations, such as increased cardiac output and stroke volume, and peripheral adaptations including increases in a range of enzymatic concentrations, in both trained an untrained individuals. ${ }^{11-15}$ It has been hypothesised that some of the adaptations to this type of training are associated with the metabolic demands and signalling responses which occur in the early stages of a sprint. Studies have therefore also investigated shorter exercise bouts to determine whether adaptations similar to those observed following 30 second sprints can be elicited. ${ }^{16-18}$

Taylor et al. ${ }^{19}$ investigated the acute effects of SIT on cell signalling responses in matched duration interval and continuous protocols, reporting no difference between the two distinct bouts of exercise despite differences in total work done, which suggests that total work is not necessarily a crucial factor when monitoring adaptations to such protocols. Chia-Lun et al. ${ }^{3}$ studied the more chronic effect of high intensity training when matched for total time. Although they reported significant improvements in $\mathrm{VO}_{2 \max }$, neither of the interval training groups were supramaximal, nor did they utilise any form of performance test. Jakeman et al. ${ }^{16}$ reported an improvement in time trial performance and time to exhaustion following 2 weeks of SIT consisting of $10 \times 6$ second sprints, with the improvements in time trial performance being associated with a prolonged time to reach the onset of blood lactate accumulation (OBLA - defined ${ }^{16}$ as a blood lactate concentration of $4 \mathrm{mmol}^{-\mathrm{L}^{-1}}$ ). Such enhancements in time trial performance have also been reported following a 30 seconds SIT programme. $^{7,20}$ While these, and other studies ${ }^{17-18}$ have reported similar adaptations, there are frequent differences between training protocols and consequently, the importance of aspects such as work:rest ratio and the duration of each individual sprint is unclear. Despite the range of SIT paradigms which have been used, there is very little information to compare very short duration $(<10 \mathrm{sec})$ sprint training directly with the more traditional $30 \mathrm{sec}$ SIT approach. It is therefore unknown if the adaptive mechanisms causing improvements in performance following SIT will generate similar adaptations with different sprint durations. Therefore, the purpose of this study was to investigate the impact of individual sprint duration on time trial performance and $\mathrm{VO}_{2 \max }$ when $\mathrm{W}: \mathrm{R}$ and total sprint duration are matched. It was hypothesised that the shorter, 6 second SIT would be at least as effective as the 30 second SIT intervention in improving these parameters. 


\section{Methods}

Thirty physically active (minimum of 5 hours week $^{-1}$ in a range of sports) males volunteered to participate in this study, which received ethical approval from the local university ethics committee, and was carried out in accordance with the declaration of Helsinki. Participants were randomly allocated, by blind draw, to one of three groups and completed either two

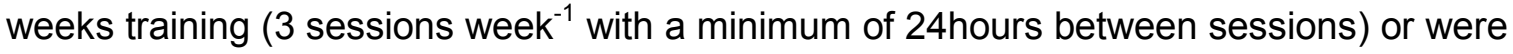
asked to follow their normal training programme. Participants in the treatment groups could continue exercising outside of the experimental conditions, however all participants were informed that this must not be exhaustive exercise. Outcome measures were a $\mathrm{VO}_{2 \max }$ test and a $10 \mathrm{~km}$ time trial; both completed on a cycle ergometer and were assessed before and after two weeks of SIT or normal training (control group).

\section{Participant characteristics}

On the first visit to the laboratory, basic anthropometric measures were taken, height was measured to the nearest $0.1 \mathrm{~cm}$ using a stadiometer (Holtain, Crosswell, Wales), weight and body composition were measured using bioelectrical impedance analysis (BIA) (Tanita, BC418MA, Amsterdam, The Netherlands) to the nearest $0.1 \mathrm{~kg}$ (table 1).

\section{Insert table 1 here}

\section{$\mathrm{VO}_{2 \max }$ test and Time Trial}

During visits one and nine, participants completed an incremental $\mathrm{VO}_{2 \max }$ test, on a Lode Excaliber cycle ergometer. Following a 5-minute warm up against a 50Watt (W) load, participants cycled against a progressively increasing resistance $(25 \mathrm{~W} \cdot \mathrm{min})$ until volitional exhaustion. During the $\mathrm{VO}_{2 \max }$ test, heart rate (Polar, FT1, England) and RPE (Borg scale 620) were recorded every minute, with respiratory variables monitored continuously (Cortex Metalyzer 3B, Leipzig, Germany). Mean $\mathrm{VO}_{2}$ during the final 30 seconds of each maximal test was recorded, and the highest value within 2SD of this mean was taken as recorded as the $\mathrm{VO}_{2 \max }$. Prior to all sessions, gas analysers were calibrated using a gas standard and the volume transducer was calibrated with a $3 \mathrm{~L}$ syringe following manufacturers guidelines.

24-48 hours following the $\mathrm{VO}_{2 \max }$ test (session 2 and 10), participants completed a selfpaced $10 \mathrm{~km}$ time trial on a Lode Excaliber cycle ergometer. The ergometer was set in linear mode, and the linear factor was calculated according to the participants' average cadence and maximum work rate from the $\mathrm{VO}_{2 \max }$ test. Participants were aware of the distance cycled but were blinded to time.

\section{Training intervention}

Volunteers in the treatment groups were randomly assigned to one of the sprint training programmes, with both set at a resistance of $7.5 \%$ of body weight on a Lode Excaliber cycle ergometer (LEM Software, Lode, The Netherlands). Both protocols used a W:R of 1:8, such that all participants completed 2 minutes of sprint work. Each volunteer completed 6 sprint sessions spread over 14 days, with a minimum of 24 hours rest in between each session. Those in the 6 sec group completed 20x6 second sprints with a 48 s recovery, and the 30 
sec group completed $4 \times 30$ second sprints with a 4-minute recovery to replicate commonly used SIT protocols. Participants were given a 10 second countdown before each sprint and were instructed to increase the cadence so they were at their maximal sprint velocity at the start of each sprint. Throughout each sprint the participants were given encouragement to ensure an 'all-out' effort. Power output was recorded throughout sprints using LEM software, at a sampling frequency of $5 \mathrm{~Hz}$. Peak power output was calculated as the highest recorded power output per sprint. Total work per session was calculated from the mean power output per sprint, multiplied by sprint time. The sum of the four or 20 sprints was then converted to kJ.

\section{Data Analysis}

Data were analysed using SPSS v21.0, and are expressed as mean \pm standard deviation, unless otherwise stated. Outcome measures were analysed using a repeated measures (RM) ANOVA. $V_{2}$ max and time trial performance were analysed using a $3 \times 2$ (group $x$ time) RM ANOVA, and power output data were analysed using a $2 \times 6$ (group $x$ time) RM ANOVA. The Mauchly sphericity test was used to assess the assumption of sphericity, with the Greenhouse-Geisser correction used for violations. Statistical significance level was set at $p \leq 0.05$, and the Scheffé post hoc test was used where appropriate. Cohens $D$ effect sizes were calculated, with $0.2,0.5$ and 0.8 being considered a small, medium or large effect size respectively. ${ }^{21}$

\section{$\underline{\text { Results }}$}

There were no significant differences between groups in participant characteristics, $10 \mathrm{~km}$ time trial, $\mathrm{VO}_{2 \max }$ and peak power output at baseline.

There was a main effect of time for time trial performance, which improved significantly in both training groups (Figure 1), by $5.1 \%$ in $6 \mathrm{sec}(d=0.31), 6.2 \%$ in $30 \mathrm{sec}(d=0.47)(p<0.05)$, but there was no change in the control group $(-1.0 \% ; p>0.05)$. An interaction effect was also observed, with post hoc analysis showing no significant difference between the $6 \mathrm{sec}$ and 30 sec groups $(p>0.05)$. There was a significant difference between the $30 \mathrm{sec}$ and control groups $(p<0.05)$, but no statistically significant difference between the $6 \mathrm{sec}$ and control groups $(p>0.05)$. There was no significant main effect for time in $\mathrm{VO}_{2 \max }$ in either intervention, and there was no group*time interaction $(p>0.05)$.

Insert Figure 1 here

Peak power output was achieved on either the first or second sprint of each training session. While there was no significant difference in peak power output between training groups $(p>0.05)$, there was a significant time and group*time interaction effect for peak power in both conditions $(p<0.05$; Figure 2a). Peak power output in $6 \mathrm{sec}$ increased significantly by $9 \%$ from session 1 to session $6(d=0.3)$, and by $20 \%$ in $30 \mathrm{sec}(d=1.2)$ from session 1 to the final sprint session (Figure 2a). There was a significant group and group*time interaction for 
total work done $(\mathrm{kJ} ; p<0.05)$, with the 6 sec group doing significantly more work than the 30 sec group ( $d=2.1$ ), however, there was no main effect of time for total work done (Figure $2 b$ ).

Insert Figure 2 here

\section{Discussion}

The main finding of this study is that both a 6 and 30 second repeated sprint intervention for 2 weeks, that were matched for total sprint time and $W: R$, resulted in similar improvement in time trial performance compared to a control group. There were, however, no effects of either training protocol on $\mathrm{VO}_{2 \max }$.

Time trial (TT) performance improved significantly in both the $30 \mathrm{sec}$ and $6 \mathrm{sec}$ training group, and remained unchanged in the control group. The improvement in performance of the $30 \mathrm{sec}$ group $(6.2 \%)$ is similar to that reported by Burgomaster et $a .^{20}$, who used the same $30 \mathrm{sec}$ training protocol, reporting a $9.6 \%$ improvement. TT performance in the $6 \mathrm{sec}$ group also improved (5.1\%). In a training study paper by Taylor et al. ${ }^{23}$ their training control group followed a similar programme as to the $30 \mathrm{sec}$ group in this current study.

Interestingly, they reported no improvement in time trial performance following the training period. The difference in the findings of this work and the current study could be firstly that their work was conducted on trained cyclists and also that the time trial distance was of a longer duration. SIT seems to be a potent method for improving performance over shorter time trials.

Although not directly measured in the current study, an increase in mitochondrial enzymes including citrate synthase activity have previously been reported following 30 second SIT protocols $s^{6,15,20,24}$, and changes such as these may have improved the oxidative potential of the muscle and subsequent exercise performance during the current study in both training groups. Although still not clear, the increased flux between rest and exercise may have caused greater perturbations to the muscle milieu during the 6 s supramaximal efforts is a possible factor for the adaptations reported following such training bouts. ${ }^{19}$ Recent work of Taylor et al. ${ }^{19}$ evaluating duration matched interval and continuous exercise demonstrated that, despite completing significantly more work in their interval training group, the magnitude of AMPK phosphorylation did not differ between groups. This work supports that of the current study in that a major determinant for adaptation stems from the ability to achieve repeated peak power outputs during the intervals rather than complete more work as demonstrated in the $6 \mathrm{~s}$ training group. It has previously been suggested that the major drivers of performance improvements may occur in the first $6-10$ seconds of SIT, with a 6 second training approach being sufficient to elicit significant performance benefits. ${ }^{16}$ While there was a slightly greater time trial improvement in the $30 \mathrm{sec}$ group, the lack of significant performance differences between groups would suggest that a 6 second protocol can be as equally beneficial method to elicit performance adaptations when matched for total sprint time, and W:R. Peak power output is typically observed within the early portion of the sprint, and as similar adaptations occurred following both sprint protocols, achieving peak power may be an important feature of performance related adaptations. ${ }^{18}$ In the current study, participants accelerated to their top speed at the start of the sprint, ensuring that peak power 
output was achieved and sustained for as long as possible. Zelt et al. ${ }^{25}$ compared a 30 and 15 second sprint, and found performance adaptations including an increased $\mathrm{VO}_{2 \max }$ and critical power, but found no difference between conditions. It may therefore be that repeatedly reaching peak power, rather than sprint duration or total work completed, is the determining factor for improvements in exercise performance. To our knowledge, this current study is the first to attempt to investigate this by controlling for the work:rest ratio in this way.

Following two weeks of SIT, peak power output per session significantly increased in both the $30 \mathrm{sec}(+20 \%)$ and the $6 \mathrm{sec}$ groups (+9\%) (Figure 2a). Burgomaster et al. ${ }^{20}$ reported an increase in peak power output by $5.4 \%$ following 6 sessions of $30 \mathrm{sec}$ sprints in 2 weeks, however the increase in the current study is more similar to that of Burgomaster et al. ${ }^{7}$, who reported a $17 \%$ increase in peak power output following 6 weeks of the $30 \mathrm{sec}$ SIT protocol. Improvement in peak power output has also been reported in studies utilizing shorter $(<10$ seconds) supramaximal bouts, including repeated 5 second and 6 second sprints ${ }^{17,16}$, which may be linked with an increase in the activity of glycolytic enzymes phosphofructokinase (PFK) and Hexokinase (Hex $)^{26-27}$ and the improved resynthesis of $\mathrm{PCr}$ during the recovery period. ${ }^{28}$ While not assessed in the current study, increases in PFK have been shown to occur as $\mathrm{pH}$ increases and accelerates the rate of glycolysis, fuelling the initial 5-10 seconds of sprinting. ${ }^{29}$ Despite the significant increase in peak power, analysis of total work done during the training sessions indicated that there was no significant change in either training group. This therefore indicates a poorer fatigue index following training, particularly in the 30 sec group, and although participants were able to achieve similar peak power outputs, these data suggest that restoration of mean power output was slower. ${ }^{28}$ In addition, those in the 6 sec group did significantly more work than those in the $30 \mathrm{sec}$ group, which is likely to reflect the fact that the shorter sprint resulted in less depletion in stored glycogen, and an ability to better resynthesize PCr needed to achieve repeated peak performance. This may also have resulted in the process of glycolysis becoming the dominant driver of exercise ${ }^{18}$, potentially increasing glycolytic enzyme activity including Hex and PFK to fuel the sprint during the latter sprints. This again may indicate that total work done is not necessarily the main driver of adaptation. It should be noted, that while the differences in work done between the two training groups was significant, individual pacing strategies may have contributed to a portion of this difference. We did not specifically look to protect against pacing, aside from giving strong verbal encouragement, and this is a potential limitation of this study, which future designs may wish to consider guarding against.

Similar to previous studies, which have implemented a 2-week SIT programme ${ }^{20}$, there was no significant change in $\mathrm{VO}_{2 \max }$ in either group. Previous research has indicated that changes in the activity of oxidative enzymes associated with improvements in $\mathrm{VO}_{2 \max }$, such as citrate synthase, can take up to 6 weeks to reach a higher steady state. ${ }^{30}$ Additionally, central adaptations that influence $\mathrm{VO}_{2 \max }$ may take longer to occur than the 2-week intervention used in the present study. ${ }^{31}$ While there were no statistically significant improvements observed in the current study, it would be of interest to further investigate responses to SIT over a more prolonged period. 
247 This study found that two-weeks of SIT comprising either a 6 or 30 second repeated bouts of 248 exercise which were matched for total sprint time and work:rest ratio elicited similar changes 249 in performance. In comparison with a control group, there were significant improvements in time trial performance, and sprint power output significantly increased for both groups. Adaptations due to the shorter sprint bout may be due to the greater amount and quality of work that can be completed during the $6 \mathrm{sec}$ protocol. This study is the first to match duration and work:rest ratio in this way, and provides interesting insight into adaptations to this type of training.

\section{Practical applications}

- Two-weeks of SIT using either a 6 or 30 second repeated bouts significantly improved athletic performance in comparison with a control group on a $10 \mathrm{~km}$ TT

265

\section{Acknowledgements}

We would like to thank the volunteers who participated in this study. No financial assistance was provided for the project, and the authors report no conflict of interest 
1. Babraj, J., Vollaard, N., Keast, C. et al. (2009). Extremely short duration high intensity interval training substantially improves insulin action in young health males. BMC Endocrine Disorders, 9(3).

2. Adamson, S., Lorimer, R., Cobley, J. et al. (2014). High intensity training improves health and physical function in middle aged adults. Biol, 3, 333-344.

3. Chia-Lun, L., Wei-Chieh, H., Ching-Feng, C. (2016). Physiological Adaptations to Sprint Interval Training with Matched Exercise Volume. Med Sci Sports Exerc, in press, DOI: 10.1249/MSS.0000000000001083.

4. Nybo, L., Sundstrup, E., Jakobsen, M.D. et al. (2010). High-Intensity Training versus Traditional Exercise Interventions for Promoting Health. Med Sci Sports Exerc. 42 (10), 1951-1958.

5. Helgerud, J., Høydal, K., Wang, E. et al. (2007). Aerobic High-Intensity Intervals Improve $\mathrm{VO}_{2 \max }$ More Than Moderate Training. Med Sci Sports Exerc. 39 (4), 665671.

6. Gibala, M.J., Little, J.P., Essen, M.V. et al. (2006). Short-term Sprint Interval versus Traditional Endurance Training: Similar Initial Adaptations in Human Skeletal Muscle and Exercise Performance. J Physiol. 575 (3), 901-911.

7. Burgomaster, K.A., Howarth, K.R., Phillips, S.M. et al. (2008). Similar Metabolic Adaptations During Exercise After Low Volume Sprint Interval and Traditional Endurance Training in Humans. J Physiol. 586 (1), 151-160.

8. Gibala, M.J. \& McGee, S.L. (2008). Metabolic Adaptations to Short-term HighIntensity Interval Training: A Little Pain for a Lot of Gain? Exerc Sport Sci Reviews. 36 (2), 58-63.

9. Bartlett, J.D., Close, G.L., Maclaren, D.P.M. et al. (2011). High-Intensity Interval Running is Perceived to be More Enjoyable than Moderate-Intensity Continuous Exercise: Implications for Exercise Adherence. J Sport Sci. 29 (6), 547-553.

10. Kong, Z., Fan, X., Sun, S. et al. (2016). Comparison of high-intensity interval training and moderate-to-vigorous continuous training for cardiometabolic health and exercise enjoyment in obese young women: A randomized controlled trial. PLoS ONE. 11(7), e0158589. doi: 10.1371/journal.pone.0158589.

11. Laursen, P.B. \& Jenkins, D.G. (2002). The Scientific Basis for High Intensity Interval Training. Sports Med. 32 (1), 53-73.

12. Bayati, M., Farzad, B., Gharakhanlou, R. et al. (2011). A Practical Model of LowVolume High-Intensity Interval Training Induces Performance and Metabolic Adaptations that Resemble 'all-out' Sprint Interval Training. J Sport Sci Med. 10 (1), 571-576. 
13. Harmer, A.R., McKenna, M.J., Sutton, J.R. et al. (2000). Skeletal Muscle Metabolic and Ionic Adaptations During Intense Exercise Following Sprint Training in Humans. J Appl Physiol. 89 (1), 1793-1803.

14. Creer, A.R., Ricard, M.D., Conlee, R.K. et al. (2004). Neural, Metabolic, and Performance Adaptations to Four Weeks of High Intensity Sprint-Interval Training in Trained Cyclists. Int J Sports Med. 25 (1), 92-98.

15. Burgomaster, K.A., Hughes, S.C., Heigenhauser, G.J.F. et al. (2005). Six Sessions of Sprint Interval Training Increases Muscle Oxidative Potential and Cycle Endurance Capacity in Humans. J Appl Physiol. 98 (1), 1985-1990.

16. Jakeman, J., Adamson, S. \& Babraj, J. (2012). Extremely short duration high intensity training substantially improves endurance performance in triathletes. Appl Physiol, Nut Metabol. 37 (5), 976-981.

17. Linossier, M.T., Denis, C., Dormois, D. et al. (1993). Ergometric and Metabolic Adaptation to a 5-s Sprint Training Programme. Eur J Physiol. 67 (1), 408-414.

18. Hazell, T.J., MacPherson, R.E.K., Gravelle, B.M.R. et al. (2010). 10 or 30-s Sprint Interval Training Bouts Enhance Both Aerobic and Anaerobic Performance. Eur J Appl Physiol Occ Physiol. 110 (1), 153-160.

19. Taylor, C.W., Ingham, S.A., Hunt, J.E.A. et al. (2016a). Exercise duration-matched interval and continuous sprint cycling induce similar increases in AMPK phosphorylation, PGC-1 $\alpha$ and VEGF mRNA expression in trained individuals. Eur $J$ Physiol, 116, 1445-1454.

20. Burgomaster, K.A., Heigenhauser, G.J.F. \& Gibala, .M.J. (2006). Effect of ShortTerm Sprint Interval Training on Human Skeletal Muscle Carbohydrate Metabolism During Exercise and Time-Trial Performance. J Appl Physiol. 100 (1), 2041-2047.

21. Cohen, J (1988). Statistical Power Analysis for the Behavioural Sci. 2nd ed. Hillsdale: $\mathrm{NJ}$ : Lawrence Erlbaum.

22. Paton, C.D. \& Hopkins, W.G. (2006). Variation in Performance of Elite Cyclists from Race to Race. Eur J Sport Sci. 6 (1), 25-31.

23. Taylor, C.W., Ingham, S.A., and Ferguson, R.A. (2016b). Acute and chronic effect of sprint interval training combined with postexercise blood-flow restriction in trained individuals. Exp Physiol, 101.1, 143-154.

24. Little, J.P., Safdar, A., Wilkin, G.P. et al. (2010). A practical model of low-volume high-intensity interval training induces mitochondrial biogenesis in human skeletal muscle: potential mechanisms. J Physiol. 588 (6), 1011-1022.

25. Zelt, J.G.E., Hankinson, P.B., Foster, W.S. et al. (2014). Reducing the Volume of Sprint Interval Training Does Not Diminish Maximal and Submaximal Performance Gains in Healthy Men. Eur J Physiol. 114 (11), 2427-2436. 
26. Rodas, G., Ventura, J.L., Cadefau, J.A. et al. (2000). A Short Training Programme for the Rapid Improvement of Both Aerobic and Anaerobic Metabolism. Eur J Physiol. 82 (5-6), 480-486.

27. MacDougall, J.D., Hicks, A.L., MacDonald, J.R. et al. (1998). Muscle Performance and Enzymatic Adaptations to Sprint Interval Training. J Appl Physiol. 84 (6), 21382142.

28. Bogdanis, G.C., Nevill, M.E., Boobis, L.H. et al. (1995). Recovery of power output and muscle metabolites following $30 \mathrm{~s}$ of maximal sprint cycling in man. $J$ Appl Physiol, 482(2), 467-480.

29. Beneke, R., Pollman, C., Bleif, I. et al. (2002). How Anaerobic is the Wingate Anaerobic Test for Humans? Eur J Physiol. 87 (4-5), 388-392.

30. Hood, D.A. (2001). Invited Review: Contractile Activity-Induced Mitochondrial Biogenesis in Skeletal Muscle. J Appl Physiol. 90 (3), 1137-1157.

31. Gist, N.H., Freese, E.C. \& Cureton, K.J. (2014). Comparison of Responses to Two High-Intensity Intermittent Exercise Protocols. J Strength Cond Res. 28 (11), 30333040.

Figure 1: 10km time trial performance; ${ }^{*}$ denotes a significant difference from baseline

Figure 2: Panel a) Peak power output; * denotes a significant increase from session 1 to session 6; Panel b) Total work done; * denotes a significant difference between groups 


\begin{tabular}{ccccc} 
Group & Age (yrs) & Height $(\mathbf{m})$ & Body Mass $(\mathbf{k g})$ & Body Fat (\%) \\
\hline $6 \sec (n=10)$ & $21 \pm 4$ & $1.78 \pm 0.06$ & $75.7 \pm 13.9$ & $14.0 \pm 5.9$ \\
$30 \sec (n=10)$ & $21 \pm 4$ & $1.84 \pm 0.06$ & $83.0 \pm 10.2$ & $14.0 \pm 3.3$ \\
$\operatorname{Con}(n=10)$ & $23 \pm 3$ & $1.82 \pm 0.07$ & $82.4 \pm 7.6$ & $14.9 \pm 3.6$ \\
\hline
\end{tabular}

Table 1. Participant anthropometric characteristics 


\begin{tabular}{|c|c|c|c|c|c|}
\hline Variable & Condition & & Pre & Post & $d$ \\
\hline \multirow{6}{*}{ TT (Seconds) } & \multirow{2}{*}{$6 \mathrm{sec}$} & $\begin{array}{c}\text { Mean } \pm \\
S D\end{array}$ & & & \multirow{2}{*}{0.31} \\
\hline & & $95 \% \mathrm{Cl}$ & $559-701$ & $541-655$ & \\
\hline & \multirow[b]{2}{*}{$30 \mathrm{sec}$} & Mean \pm & $579 \pm 68$ & $543 \pm 85^{*}$ & \multirow[b]{2}{*}{0.47} \\
\hline & & $95 \% \mathrm{Cl}$ & $537-621$ & $490-596$ & \\
\hline & \multirow{2}{*}{ Con } & Mean \pm & $631 \pm 104$ & $634 \pm 99$ & \multirow{2}{*}{0.03} \\
\hline & & $95 \% \mathrm{Cl}$ & $567-695$ & $573-695$ & \\
\hline \multirow{6}{*}{$\mathrm{VO}_{2 \max }\left(\mathrm{ml} \cdot \mathrm{kg} \cdot \mathrm{min}^{-1}\right)$} & \multirow{2}{*}{$6 \mathrm{sec}$} & $\begin{array}{c}\text { Mean } \pm \\
S D\end{array}$ & $57 \pm 8$ & $59 \pm 10$ & \multirow{2}{*}{0.22} \\
\hline & & $95 \% \mathrm{Cl}$ & $52-62$ & $53-65$ & \\
\hline & \multirow[b]{2}{*}{$30 \mathrm{sec}$} & $\underset{S D}{M} \pm$ & $57 \pm 6$ & $58 \pm 9$ & \multirow[b]{2}{*}{0.13} \\
\hline & & $95 \% \mathrm{Cl}$ & $53-61$ & $52-64$ & \\
\hline & \multirow{2}{*}{ Con } & $\begin{array}{c}\text { Mean } \pm \\
\text { SD }\end{array}$ & $52 \pm 9$ & $52 \pm 6$ & \multirow{2}{*}{0.13} \\
\hline & & $95 \% \mathrm{Cl}$ & $47-59$ & $48-56$ & \\
\hline
\end{tabular}

Table 2: Time trial and $\mathrm{VO}_{2 \max }$ data

*Denotes a significant difference from baseline $(p<0.05)$. 


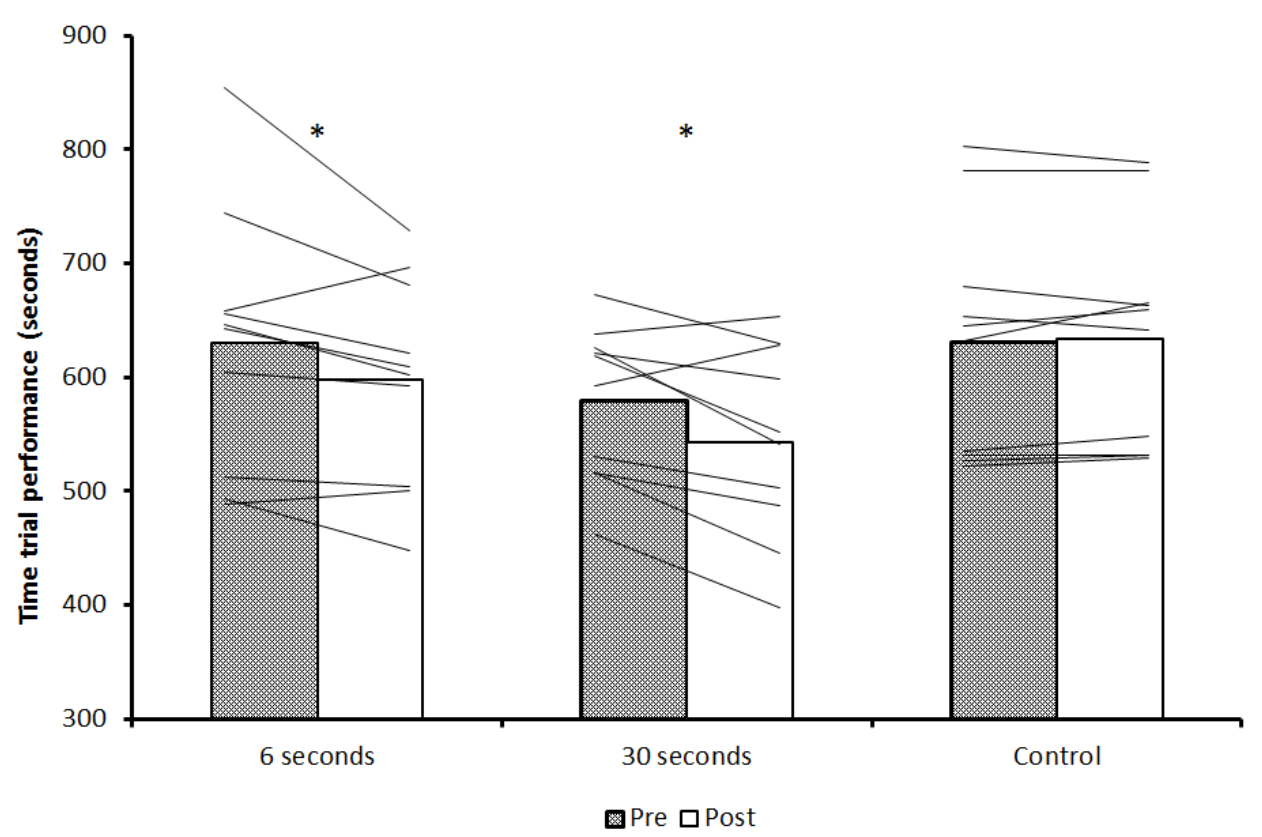

Fig $1.10 \mathrm{~km}$ time trial performance; * denotes a significant difference from baseline.
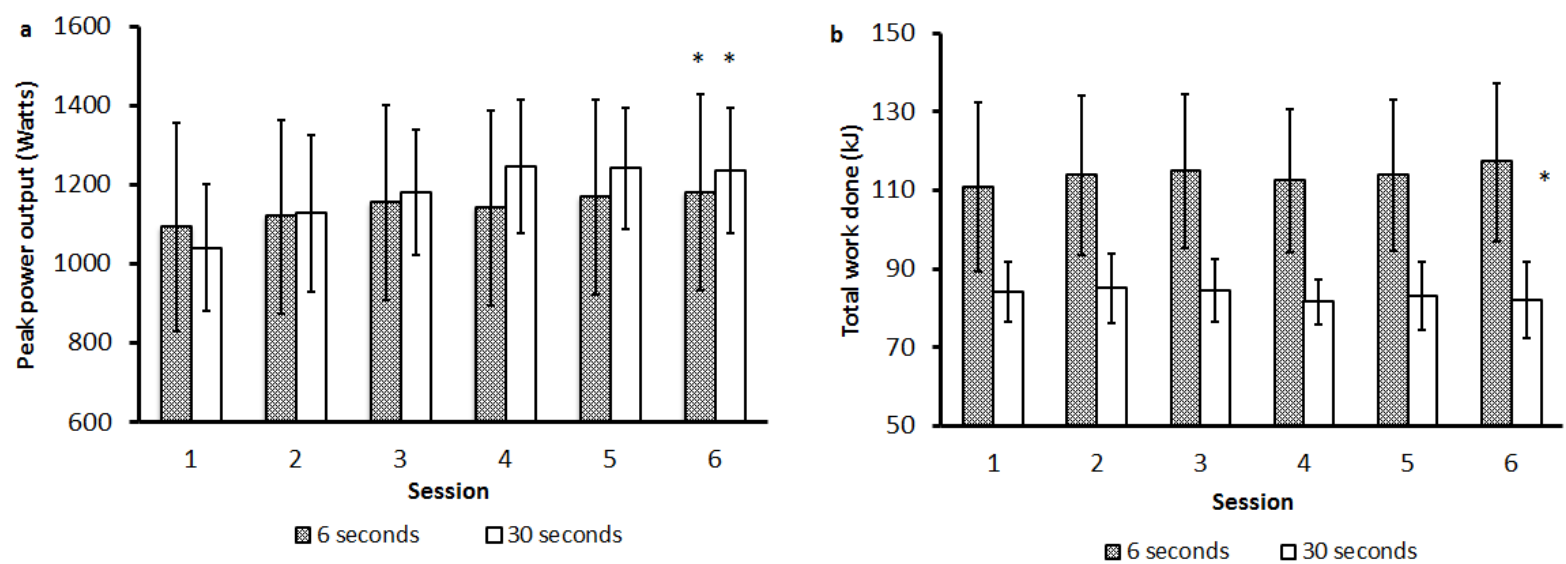

Fig 2. Panel (a) Peak power output; * denotes a significant increase from session 1 to session 6; Panel (b) Total work done; ${ }^{*}$ denotes a significant difference between groups. 